\title{
ON INEQUALITIES OF WEAK TYPE
}

\author{
BY S. SAWYER
}

Communicated by P. R. Halmos, March 3, 1964

1. Introduction. E. M. Stein [3] has recently established a general principle for obtaining inequalities satisfied by sequences of operators. Suppose that for finite measure space $X$ one has a sequence of continuous linear transformations $\left\{T_{n}\right\}$ on $L^{p}(X)$ into $L^{p}(X)$, where $1 \leqq p \leqq 2$, and that further

$$
\lim _{n \rightarrow \infty} T_{n} f(x)
$$

exists a.e. for every $f(x)$ in $L^{p}(X)$. Then, under the assumption that $X$ is a compact group ${ }^{1}$ and the $\left\{T_{n}\right\}$ translation invariant, Stein was able to predict the existence of a constant $\Omega_{0}$ such that

$$
m\left[\left\{x: \sup _{1 \leqq k<\infty}\left|T_{k} f(x)\right| \geqq A\right\}\right] \leqq \frac{\Omega_{0}}{A^{p}} \int_{X}|f(x)|^{p} d x
$$

for every $f(x)$ in $L^{p}(X)$ and $A>0$. Conclusions of this sort are called inequalities of weak type, or, where $T^{*} f(x)=\sup _{n}\left|T_{n} f(x)\right|=T^{*}(x, f)$, that the operator $T^{*}$ is of weak type $(p, p)$. Inequality (1) has of ten appeared with convergence a.e. in analysis, but a single result of this generality, predicting (1) as a consequence of convergence, had never before been obtained. Indeed, it seems to contain most of the known examples of (1) in Fourier analysis as special cases. However, the general idea, that convergence alone might yield (1), seems to crop up in situations much removed from group theoretical considerations. For example, such inequalities are of ten the first step in proving theorems in probability and ergodic theory; it would be interesting to know to what extent they are implicit in the statement of their results as well. Using the general techniques of Stein and of ergodic theory, we have succeeded in proving an extension of this principle which includes many of these other results, as well as in throwing new light on old ones. Our results will only be stated here; complete details, together with other theorems of a similar nature, will appear elsewhere.

2. Definitions and preliminaries. Assume $w(x)$ is a measure-preserving transformation of a finite measure space $(X, \mathcal{L}, m)$, and let $R_{n}$.

${ }^{1}$ Or a homogeneous space of a compact group, as in rotations on the unit sphere in 
$\left\{T_{n}\right\}$ be as before. Then, $\left\{T_{n}\right\}$ is said to commute with $w(x)$ if for every $f(x)$ in $L^{p}(X)$ and $g(x)=f(w(x)), T^{*}(w(x), f) \leqq T^{*}(x, g)$ a.e. (Notice this is actually a condition on $T^{*}$.) For example, let $X$ be the unit interval, and $T_{n} f(x)$ the $n$th partial sum of the expansion of $f(x)$ in terms of Haar functions. Then, the sequence $\left\{T_{n}\right\}$ commutes with the ergodic transformation $w(x)=2 x(\bmod 1)$, although, in general, $T_{n} f(w(x))$ is different from $T_{n} g(x)$. (See Example 3 for a similar situation.)

If $\mathcal{F}$ is a collection of measure-preserving transformations on $X$, it will be called an ergodic family (on $X$ ) if for any two sets $A, B$ in $\&$ with $m(A)>0, m(B)>0$, there exists $w(x)$ in $\mathcal{F}$ such that $m\left(A \cap w^{-1}(B)\right)>0$. If $\mathscr{F}$ is closed under composition, an equivalent formulation would be that if any set $F$ in $\mathcal{L}$ is fixed (i.e. $w^{-1}(F)=F$ essentially) by every $w$ in $F$, then either $m(F)=0$ or $m(X-F)=0$. Finally, if $\left\{T_{n}\right\}$ commutes with every member of some ergodic family on $X$, it will be said to be distributive.

The condition of distributiveness on $\left\{T_{n}\right\}$ is a condition to the effect that it cannot discriminate for or against any part of $X$ and, as the results of the next section will indicate, this forces it to behave in a very regular manner. Examples of distributive sequences would be any sequence of operators commuting with a given ergodic transformation, or translation invariant sequences on a compact group.

\section{Main results.}

Theorem. Assume $\left\{T_{n}\right\}$ is a distributive sequence on $X$, and that

$$
\lim _{n \rightarrow \infty} T_{n} f(x)
$$

exists a.e. for each $f(x)$ in $L^{p}(X)$. Then, $T^{*}$ is of weak type $(p, p)$.

As in the case of Stein, one can get by with less.

Corollary. Assume $\left\{T_{n}\right\}$ is distributive. Then, if $T^{*}$ is not of weak type $(p, p), T^{*} f(x)=\infty$ a.e. for every $f(x)$ in $L^{p}(X)$, with the exception of $a$ set of the first category.

For example, if $\left\{T_{n}\right\}$ is a translation invariant sequence on the unit circle, it could be considered distributive either because it commutes with a preassigned ergodic (irrational) translation of the unit circle, or because of the group theoretical structure of the circle itself. The key fact is that it commutes with too many transformations to misbehave in a moderate manner.

REMARK. In virtually every application, one can extend the con- 
clusions of these considerations in the following manner. Assume that $T^{*}$ is of weak type $(p, p)$, and that for every $f(x)$ in a dense subset of $L^{p}(X)$,

$$
m\left[\left\{x: T^{*} f(x) \geqq A\right\}\right]=\sigma\left(\frac{1}{A^{p}}\right) \quad \text { as } A \rightarrow \infty .
$$

Then, (2) holds for every $f(x)$ in $L^{p}(X)$.

4. Examples. (1) Let $C f(x)=\tilde{f}(x)$ be the function conjugate to $f(x)$ in $L^{1}(0,2 \pi)$. It was first proven by Kolmogorov [1] in 1923 that the operator $C$ is of weak type $(1,1)$; i.e. satisfies inequality (1) with $p=1, T_{n} \equiv C$. Five years later Titchmarsh [4], as a corollary to a real variable proof of this fact, obtained in addition that for any individual $f(x)$ in $L^{1}(0,2 \pi)$,

$$
m[\{x:|\tilde{f}| \geqq A\}]=\sigma\left(\frac{1}{A}\right) .
$$

Given the Abel or $(C, 1)$ summability a.e. of the conjugate series, the first result follows from Stein and the second from the remark. Thus, both results can be obtained from general principles.

(2) Let $w(x)$ be a measure-preserving transformation which commutes with every member of some ergodic family on $X$, for example a power of an ergodic transformation. Then, for an infinite matrix $\left\{u_{n k}\right\}$ and any $f(x)$ in $L^{1}(X)$, define

$$
T_{n} f(x)=u_{n 0} f(x)+u_{n 1} f(w(x))+\cdots+u_{n n} f\left(w^{n}(x)\right) .
$$

Suppose that we were given that for every $f(x)$ in a set of the second category in $L^{1}(X)$, lim sup $\left|T_{n} f(x)\right|<\infty$ a.e. Then, it would follow from the corollary that $T^{*}$ was of weak type $(p, p)$ for every $p$, $1 \leqq p \leqq 2$. Inequalities such as

$$
\begin{aligned}
& \int T^{*} f(x)^{p} d x \leqq A_{p} \int|f(x)|^{p} d x, \quad 1<p<2 \\
& \int T^{*} f(x) d x \leqq A_{1} \int|f| \log ^{+}|f| d x+A_{2}
\end{aligned}
$$

would then follow from interpolation theorems [5, Volume II, pp. 111-119]. In particular, if $\lim T_{n} f(x)$ always exists a.e., the limit function would also satisfy these inequalities. If we knew in addition that $\sum_{0}^{n}\left|u_{n k}\right| \leqq 1$ for all $n$, we could conclude in addition that for any individual $f(x)$ in $L^{p}(X)$, 


$$
m\left[\left\{x: T^{*} f(x) \geqq A\right\}\right]=\sigma\left(\frac{1}{A^{p}}\right) .
$$

If we set $u_{n k}=1 /(n+1)$, all of this information would follow directly from the Birkhoff Ergodic Theorem; indeed, inequality (4) could imply that a maximal ergodic theorem is involved in all theorems of this type.

(3) The final example is from probability. Let $\left\{x_{n}\right\}$ be a sequence of identically distributed independent random variables on a probability space $(X, \&, P)$. Assume for convenience that they have been normalized to have mean zero and variance one, and are bounded in absolute value by one. Then, for any $f$ in $L^{2}(X)$, we can define new random variables

$$
s_{n}(f)=\sum_{1}^{n} x_{k} E\left(f x_{k}\right) .
$$

By the Consistency Principle [2], it is sufficient to assume $X=\prod_{1}^{\infty}[-1,1], \mathcal{L}$ is the Borel field of $X$, and $\left\{x_{n}\right\}$ are the coordinate functions of $X$. But then the sequence $\left\{s_{n}\right\}$ commutes with the unilateral shift on $X$, and is thus distributive. In other words, the theorem of Kolmogorov that

$$
\sum_{1}^{\infty} a_{n} x_{n}
$$

converges a.e. if and only if $\sum a_{n}^{2}<\infty$ directly implies the inequality

$$
P\left(s^{*}(f) \geqq A\right) \leqq \frac{\Omega_{0}}{A^{2}} E\left(f^{2}\right),
$$

also due to Kolmogorov. If, as in the case of Rademacher functions on the unit interval, we know that the expansion of any $L^{p}$ function $(p>1)$ converges a.e., we obtain in addition that

$$
P\left(s^{*}(f) \geqq A\right) \leqq \frac{\Omega_{p}}{A^{p}} E\left(|f|^{p}\right), \quad 1<p \leqq 2,
$$

where $s^{*}$ is as usual.

Added in proof. An extension of Stein's results in another direction has recently been obtained by D. L. Burkholder [6].

\section{REFERENCES}

1. A. Kolmogorov, Sur les fonctions harmoniques conjugués et des séries de Fourier, Fund. Math. 7 (1925), 24-29. 
2. M. Loève, Probability theory, 3rd ed., Van Nostrand, Princeton, N. J., 1962.

3. E. M. Stein, On limits of sequences of operators, Ann. of Math. (2) 74 (1961), 140-170.

4. E. C. Titchmarsh, On conjugate functions, Proc. London Math. Soc. 29 (1929), 49-80.

5. A. Zygmund, Trigonometric series, Vols. 1, 2, 2nd ed., Cambridge Univ. Press, Cambridge, 1959.

6. D. L. Burkholder, Maximal inequalities as necessary conditions for almost everywhere convergence, $Z$. Wahrscheinlichkeitstheorie (to appear).

California Institute of Technology 